\title{
Filtering method for nonlinear systems with constraints
}

\author{
L.-S. Wang, Y.-T. Chiang and F.-R. Chang
}

\begin{abstract}
A constrained filtering method is proposed to deal with the filtering problems for nonlinear systems with constraints. The problem is converted to a sequence of recursive estimation problems in which the system equations and constraint conditions are treated as pscudo-measurements. To resolve the singularity problem arising from the constraints, a modified maximum-likelihood method for nonlinear systems is developed. The simulation results from the application of the proposed scheme to the target tracking problem shows that the constrained filtering method can enhance the performance of filter design significantly.
\end{abstract}

\section{Introduction}

Constraints naturally arise for complex physical systems. Those constraints may be inherent, such as the conservation of energy of a mechanical system, or supplemental, such as the motion of a vehicle on a surface. To deal with the filtering problem of a nonlinear system with constraints, the condition of constraints must be appropriately accommodated. While it is necessary to take the supplemental constraints into consideration in the filter design to acquire meaningful results, the inclusion of inherent constraints in the design may enhance the performance of the filter. However, singularity problems often appear in the treatment of the constraint conditions. For linear systems, the conditions may be incorporated into the framework of a singular system or a descriptor system $[1,2]$, and the filtering method developed in [3] may be applied. Since in most applications nonlinearity inevitably occurs, it is desirable to develop a filtering method for nonlinear system with constraints. The methodology developed in this paper provides a solution to suit this need.

The proposed scheme is a recursive estimation method that deals with system equations, measurement equations, and constraint conditions simultaneously. Similar to [3], the idea is to convert the filtering problem into recursive estimation problems in which both system equations and constraint conditions are treated as pseudo-measurements. While the corresponding measurement equations from the former may be corrupted by the process disturbances, those from the latter are associated with the zero error

\section{(C) IEE, 2002}

IEE Proceedings online no. 20020799

DOI: 10.1049 /ip-cta:20020799

Paper first received 16th January and in revised form 1st October 2002

L.-S. Wang is with the Institute of Applied Mechanics, National Taiwan University, Taipei, Taiwan

Y.-T. Chiang is with the Department of Electrical Engineering. Lee-Ming Institute of Technology, Taipei, Taiwan

F.-R. Chang is with the Department of Electrical Engineering, National Taiwan University, Taipci, Taiwan covariance matrix. Due to the existence of the singular covariance matrix, the classical maximum likelihood method nceds to be modified. By appropriately choosing the cost function, the method of the Lagrange multiplier is applied to derive the equations of the optimal solution. Since the underlying system is nonlinear, the equations to be solved are also nonlinear. With properly selected initial conditions, the Newton-Raphson method is then used to efficiently obtain the solution. The constrained filtering method is fulfilled by recursively carrying out the iterative process.

The treatment of constraint conditions as pseudomeasurements has been discussed in $[4,5]$ and [6] to deal with the constraints rclated to the problems of target tracking and locating objects by sensors, respectively. In $[4,5]$, the constraint of constant speed of the target (an inherent constraint) is realised by a linearised soft pseudomeasurement with the inclusion of a random variable such that the error covariance matrix is not singular. On the other hand, in [6] the so-called strong (i.e. hard) geometric constraints of the object (which is a supplemental constraint) is treated by a sequence of weak constraints for which the error covariance matrices are also nonsingular. In contrast, the method proposed in this paper attacks the problem of singularity arising from the constraint directly. Both soft and hard constraints can be simultancously managed by our algorithm. A similar method has been used in [7] to deal with the hard constraint arising in the attitude determination problem.

To illustrate the application of the proposed scheme, the target tracking problem as discussed in $[4,5]$ is considered further in this paper. The target is assumed to move in a circular orbit with constant speed. The tracking radar can provide the measurement of the range, the azimuth, and the elevation of the moving target. The problem may be formulated in three different forms with the constraints being treated as inherent, supplemental, and soft, respectively. For all these cases, it is shown that the proposed method performs better than the plain extended Kalman filter (EKF) method and the method of EKF with constraint presented in [4]. Additional information on the characteristics of the system is indeed valuable in the filter design. Other types of constraint or information such as the flight 
envelope discussed in [8] may exist and other approaches, such as the particle filter or the projection technique, may be adopted [9]. The methodology presented here provides an effective tool to solve the filtering problems with hard or soft constraints efficiently.

\section{Maximum-likelihood method}

In this Section, the classical maximum-likelihood method is first reviewed. However, to deal with the singularity problem associated with the inclusion of constraint conditions, the method must be modified. The basic idea has been discussed in [3], and the scheme developed below is used to tackle the nonlinear problems.

\subsection{Maximum likelihood estimate for problems with nonsingular covariance matrix}

Consider an algebraic estimation problem

$$
\mathbf{y}=\mathbf{H x}+\xi \text {, }
$$

where $\mathbf{x}$ is an unknown $n$-dimensional vector, $\mathbf{y}$ is a $p(\geq n)$ dimensional measurement vector, $\mathbf{H}$ is the $p \times n$ matrix mapping $\mathbf{x}$ to $\mathbf{y}$, and $\xi$ is a zero-mean Gaussian random vector with covariance matrix $\mathbf{S}$. If the matrix $\mathbf{S}$ is positivedefinite, the problem of finding the maximum likelihood estimate that maximises the likelihood function of $\xi$ is equivalent to the weighted least squares problem, in which the cost function

$$
J=\frac{1}{2} \xi^{T} \mathbf{S}^{-1} \xi
$$

is minimised subject to the linear constraint (1) with the measurement $\mathbf{y}$. The problem is readily solved to yield the maximum likelihood estimate of $\mathbf{x}$ as

$$
\hat{\mathbf{x}}=\left(\mathbf{H}^{T} \mathbf{S}^{-1} \mathbf{H}\right)^{+} \mathbf{H}^{T} \mathbf{S}^{-1} \mathbf{y}
$$

with error covariance matrix

$$
\mathbf{P}=\left(\mathbf{H}^{T} \mathbf{S}^{-1} \mathbf{H}\right)^{+}
$$

In the previous equations, the notation of pseudo-inverse $[10,11]$ has been adopted.

\subsection{Maximum likelihood estimate for nonlinear problems with singular covariance matrix}

If now the $(p \times p)$ error covariance matrix $\mathbf{S}$ is singular, or positive semi-definite, the cost function defined in $(2)$ is not well defined, and the classical maximum-likelihood method discussed above must be modified. Let $\boldsymbol{\Gamma}$ be the full-column-rank $(p \times r)$ square root of $\mathbf{S}$ such that $\mathbf{S}=\boldsymbol{\Gamma} \boldsymbol{\Gamma}^{T}$, where $r$ denotes the rank of $\mathbf{S}$. The error vector $\xi$ may be then generated by a zero-mean Gaussian random vector $\eta$ of dimension $r$ with the identity covariance matrix $\mathbf{I}$, through a prefilter with transformation matrix $\boldsymbol{\Gamma}$, i.e. $\zeta=\Gamma \eta$. Furthermore, if the problem is nonlinear, (1) may be rewritten as

$$
\mathbf{y}=\mathbf{h}(\mathbf{x})+\xi=\mathbf{h}(\mathbf{x})+\mathbf{\Gamma} \eta
$$

The associated maximum-likelihood estimate problem is now cast to be equivalent to the least squares problem of minimising

$$
J=\frac{1}{2} \eta^{T} \eta
$$

subject to the nonlinear constraint (5) on $\mathbf{x}$ and $\eta$.

526
With the Lagrange multiplier vector $\lambda$, the previous constrained minimisation problem can be solved by finding the critical condition of the Lagrangian

$$
L(\eta, \mathbf{x}, \lambda)=\frac{1}{2} \eta^{T} \eta+\lambda^{T}(\mathbf{y}-\mathbf{h}(\mathbf{x})-\mathbf{\Gamma} \eta)
$$

which gives rise to the constraint condition (5) and the following equations

$$
\begin{array}{r}
\eta-\Gamma^{T} \lambda=\mathbf{0} \\
\mathbf{H}(\mathbf{x})^{T} \lambda=\mathbf{0}
\end{array}
$$

where $\mathbf{H}(\mathbf{x})=\partial \mathbf{h} / \partial \mathbf{x}(\mathbf{x})$. Substituting the expression of $\eta$ in (8) into (5), we obtain

$$
\mathbf{h}(\mathbf{x})+\mathbf{S} \lambda=\mathbf{y}
$$

The optimal estimate is then obtained by solving the previous equation and (9) simultaneously for $\mathbf{x}$ and $\lambda$.

In particular, if the problem is linear, we have $\mathbf{h}(\mathbf{x})=\mathbf{H x}$, and the equations to be solved become

$$
\left[\begin{array}{cc}
\mathbf{S} & \mathbf{H} \\
\mathbf{H}^{T} & \mathbf{0}
\end{array}\right]\left[\begin{array}{l}
\lambda \\
\mathbf{x}
\end{array}\right]=\left[\begin{array}{l}
\mathbf{y} \\
\mathbf{0}
\end{array}\right]
$$

After finding the pseudo-inverse of the block matrix consisting of $\mathbf{S}$ and $\mathbf{H}$ in the left-hand side of the previous equation, the maximum likelihood estimate of $\mathbf{x}$ is given by

$$
\hat{\mathbf{x}}=\left[\begin{array}{ll}
\mathbf{0} & \mathbf{I}
\end{array}\right]\left[\begin{array}{cc}
\mathbf{S} & \mathbf{H} \\
\mathbf{H}^{T} & \mathbf{0}
\end{array}\right]^{+}\left[\begin{array}{l}
\mathbf{1} \\
\mathbf{0}
\end{array}\right] \mathbf{y}
$$

and the corresponding error covariance matrix $\mathbf{P}=$ $E\left[(\mathbf{x}-\hat{\mathbf{x}})(\mathbf{x}-\hat{\mathbf{x}})^{\mathrm{T}}\right]$ can be obtained as

$$
\mathbf{P}=-\left[\begin{array}{ll}
\mathbf{0} & \mathbf{I}
\end{array}\right]\left[\begin{array}{cc}
\mathbf{S} & \mathbf{H} \\
\mathbf{H}^{T} & \mathbf{0}
\end{array}\right]^{+}\left[\begin{array}{l}
\mathbf{0} \\
\mathbf{I}
\end{array}\right] .
$$

If the block square matrix is invertible, for which the condition is given in [3], the pseudo-inverse becomes the true inverse, which occurs when there are no redundant perfect measurements.

On the other hand, if the problem is nonlinear and a prior estimate $\mathbf{x}^{-}$is given, the set of equations (9) and (10) may be solved by using the Newton-Raphson iteration method. With the prior estimate, we apply the technique discussed above for linear equation to the linearised equations of $(9)$ and (10) about $\mathbf{x}^{-}$in the form of

$$
\begin{aligned}
\mathbf{H}\left(\mathbf{x}^{-}\right)^{T} \lambda & =\mathbf{0} \\
\mathbf{H}\left(\mathbf{x}^{-}\right) \mathbf{x}+\mathbf{S} \lambda & =\mathbf{y}-\mathbf{h}\left(\mathbf{x}^{-}\right)+\mathbf{H}\left(\mathbf{x}^{-}\right) \mathbf{x}^{-}
\end{aligned}
$$

The initial condition for the Newton-Raphson method is then given by

$$
\left[\begin{array}{l}
\hat{\lambda}_{0} \\
\mathbf{x}_{0}
\end{array}\right]=\left[\begin{array}{cc}
\mathbf{S} & \mathbf{H}\left(\mathbf{x}^{-}\right) \\
\mathbf{H}^{T}\left(\mathbf{x}^{-}\right) & \mathbf{0}
\end{array}\right]^{+}\left[\begin{array}{c}
\mathbf{y}-\mathbf{h}\left(\mathbf{x}^{-}\right)+\mathbf{H}\left(\mathbf{x}^{-}\right) \mathbf{x}^{-} \\
\mathbf{0}
\end{array}\right]
$$

with the initial error covariance matrix $\mathbf{P}_{0}$ being obtained from (13) with $\mathbf{H}$ being replaced by $\mathbf{H}\left(\mathbf{x}^{-}\right)$.

The iteration to find the solution of $(9)$ and $(10)$ then proceeds as follows, $j=0,1,2, \ldots$

$$
\left[\begin{array}{c}
\lambda_{j+1} \\
\mathbf{x}_{j+1}
\end{array}\right]=\left[\begin{array}{c}
\hat{i}_{j} \\
\mathbf{x}_{j}
\end{array}\right]+\mathbf{U}_{j}^{+}\left[\begin{array}{c}
-\mathbf{H}^{T}\left(\mathbf{x}_{j}\right) \lambda_{j} \\
-\mathbf{h}\left(\mathbf{x}_{j}\right)-\mathbf{S} \lambda_{j}+\mathbf{y}
\end{array}\right]
$$

where

$$
\mathbf{U}_{j}=\left[\left.\begin{array}{cc}
\mathbf{H}^{T}\left(\mathbf{x}_{j}\right) & \left(\frac{\partial}{\partial x} \mathbf{H}^{T}(\mathbf{x})\right) \\
\mathbf{S} & \mathbf{H}\left(\mathbf{x}_{j}\right)
\end{array}\right|_{\left(i_{j}, \mathbf{x}_{j}\right)}\right]
$$


Once the iterations converge at $(j+1)$ step, the maximumlikelihood estimate $\hat{\mathbf{x}}$ is given by $\mathbf{x}_{j+1}$. The corresponding covariance matrix for $\hat{\mathbf{x}}$ is derived by performing the following iterative process:

$$
\begin{aligned}
\mathbf{P}_{j+1} & \equiv E\left[\left(\mathbf{x}-\mathbf{x}_{j+1}\right)\left(\mathbf{x}-\mathbf{x}_{j+1}\right)^{T}\right] \\
& =\mathbf{P}_{j}-\mathbf{P}_{1, j}-\mathbf{P}_{1, j}^{T}+\mathbf{P}_{2, j}
\end{aligned}
$$

where

$$
\mathbf{P}_{1, j}=E\left[\left(\mathbf{x}_{j+1}-\mathbf{x}_{j}\right)\left(\mathbf{x}-\mathbf{x}_{j}\right)^{T}\right]=\left[\begin{array}{ll}
0 & \mathbf{I}
\end{array}\right] \mathbf{U}_{j}^{+}\left[\begin{array}{c}
0 \\
\mathbf{H}\left(\mathbf{x}_{j}\right) \mathbf{P}_{j}
\end{array}\right]
$$

and

$$
\begin{aligned}
\mathbf{P}_{2, j}= & E\left[\left(\mathbf{x}_{j+1}-\mathbf{x}_{j}\right)\left(\mathbf{x}_{j+1}-\mathbf{x}_{j}\right)^{T}\right] \\
= & {\left[\begin{array}{ll}
0 & \mathbf{I}
\end{array}\right] \mathbf{U}_{j}^{+} } \\
& \times\left[\begin{array}{cc}
\mathbf{H}^{T}\left(\mathbf{x}_{j}\right) \lambda_{j} \lambda_{j}^{T} \mathbf{H}\left(\mathbf{x}_{j}\right) & \mathbf{H}^{T}\left(\mathbf{x}_{j}\right) \lambda_{j} \lambda_{j}^{T} \mathbf{S}^{T} \\
\mathbf{S} \lambda_{j} \lambda_{j}^{T} \mathbf{H}\left(\mathbf{x}_{j}\right) & \mathbf{H}\left(\mathbf{x}_{j}\right) \mathbf{P}_{j} \mathbf{H}^{T}\left(\mathbf{x}_{j}\right)+\mathbf{S}+\mathbf{S} \lambda_{j} \lambda_{j}^{T} \mathbf{S}^{T}
\end{array}\right] \\
& \times\left(\mathbf{U}_{j}^{+}\right)^{T}\left[\begin{array}{l}
0 \\
\mathbf{I}
\end{array}\right]
\end{aligned}
$$

With the prior estimate being suitably chosen, which is the case for the constrained filtering method presented in the next Section, the estimate $\hat{\mathbf{x}}$, as well as the error covariance matrix $\hat{\mathbf{P}}$, can be obtained within the steps in the Newton-Raphson iteration. This method shall be termed the modified maximum-likelihood method for estimation problems.

\section{Constrained filtering method}

Based on the modified maximum-likelihood method described in the previous Section, the constrained filtering method for linear or nonlinear systems subject to constraints is constructed in this Section. The design of the linear filter may be used in the determination of the prior estimate in the development of the nonlinear filter.

\subsection{Filtering method for linear system with constraints}

First, we consider the filter design for linear systems with constraints. The problem is to find a filtering algorithm for a linear discrete dynamical system modelled by

$$
\mathbf{x}_{k+1}=\mathbf{A}_{k} \mathbf{x}_{k}+\mathbf{w}_{k}
$$

with measurement equation

$$
\mathbf{z}_{k}=\mathbf{C}_{k} \mathbf{x}_{k}+v_{k}
$$

while the state $\mathbf{x}_{k}$ is subject to the following linear constraint condition

$$
\mathbf{L}_{k} \mathbf{x}_{k}=0
$$

The process noise $\mathbf{w}_{k}$ and the measurement noise $v_{k}$ are assumed to be independent zero-mean Gaussian random processes with covariance matrices $\mathbf{Q}_{k}$ and $\mathbf{R}_{k}$, respectively. Starting with the prior estimate $\hat{\mathbf{x}}_{0}$ and the initial state error covariance matrix $\mathbf{P}_{0}$, at each step of $k=0,1$, $2, \ldots$, we want to find the estimate $\hat{\mathbf{x}}_{k+1}$ based on the previous estimate $\hat{\mathbf{x}}_{k}$ with error covariance matrix $\mathbf{P}_{k}$ and the new measurement $\mathbf{z}_{k+1}$, with the constraint condition (24) being satisfied.

For linear systems, it can be shown that the state error vector $\tilde{\boldsymbol{x}}_{k}=\mathbf{x}_{k}-\hat{\mathbf{x}}_{k}$ is a Gaussian random vector with zero mean and covariance matrix $\mathbf{P}_{k}$, which is independent of $\mathbf{w}_{k}$. In terms of $\tilde{\boldsymbol{x}}_{k},(22)$ can be expressed as

$$
\mathbf{A}_{k} \hat{\mathbf{x}}_{k}=\mathbf{x}_{k+1}-\mathbf{A}_{k} \tilde{\mathbf{x}}_{k}-\mathbf{w}_{k}
$$

Similar to the idea discussed in [3], the previous equation and the constraint condition (24) are treated as pseudomeasurements with covariance matrices $\mathbf{A}_{k} \mathbf{P}_{k} \mathbf{A}_{k}^{T}+\mathbf{Q}_{k}$ and $\mathbf{0}$, respectively. The filtering problem is then formulated as a set of recursive estimation problems, with each being modelled by (1) with components

$$
\mathbf{y}=\left[\begin{array}{c}
\mathbf{A}_{k} \hat{\mathbf{x}}_{k} \\
\mathbf{z}_{k+1} \\
0
\end{array}\right], \quad \mathbf{H}=\left[\begin{array}{c}
\mathbf{I} \\
\mathbf{C}_{k+1} \\
\mathbf{L}_{k+1}
\end{array}\right], \quad \xi=\left[\begin{array}{c}
-\mathbf{A}_{k} \tilde{\mathbf{x}}_{k}-\mathbf{w}_{k} \\
v_{k+1} \\
0
\end{array}\right]
$$

The covariance matrix for the disturbance $\xi$ is found to be

$$
\mathbf{S}_{k+1}=\left[\begin{array}{ccc}
\mathbf{A}_{k} \mathbf{P}_{k} \mathbf{A}_{k}^{T}+\mathbf{Q}_{k} & \mathbf{0} & \mathbf{0} \\
\mathbf{0} & \mathbf{R}_{k+1} & \mathbf{0} \\
\mathbf{0} & \mathbf{0} & \mathbf{0}
\end{array}\right]
$$

which is seen to be a positive-semi-definite matrix. As a result, the technique described in Section 2.1 is not applicable. Instead, we should use (12) and (13), with the substitution of $\mathbf{H}$ and $\mathbf{S}$ by (26) and (27), respectively, to obtain the maximum-likelihood estimate $\hat{\mathbf{x}}_{k+1}$, and the associated error covariance matrix $\mathbf{P}_{k+1}$.

\subsection{Filtering method for nonlinear system with hard constraints}

In the application of the constrained filtering method to many physical problems, nonlinear effects frequently arise. The techniques developed in the previous Subsection have been applied in [12] to solve the estimation problems for attitude determination, with linearised dynamical equation and constraint condition. However, to attack the nonlinearity directly, the method should be extended.

Let the nonlinear system with measurements and constraints be expressed as

$$
\begin{aligned}
\mathbf{x}_{k+1} & =\mathbf{f}_{k}\left(\mathbf{x}_{k}\right)+\mathbf{w}_{k} \\
\mathbf{z}_{k} & =\Phi_{k}\left(\mathbf{x}_{k}\right)+v_{k} \\
0 & =\mathbf{I}_{k}\left(\mathbf{x}_{k}\right)
\end{aligned}
$$

Analogous to the problem stated in the previous Subsection, we want to find $\hat{\mathbf{x}}_{k+1}, \hat{\mathbf{P}}_{k+1}$ from the estimate $\hat{\mathbf{x}}_{k}$ with error covariance matrix $\mathbf{P}_{k}$, and the measurement $\mathbf{z}_{k+1}$, at each step. Again, the state error vector $\tilde{\mathbf{x}}_{k}=\mathbf{x}_{k}-\hat{\mathbf{x}}_{k}$ is used to assess the difference between $\mathbf{f}_{k}\left(\mathbf{x}_{k}\right)$ and $\mathbf{f}_{k}\left(\hat{\mathbf{x}}_{k}\right)$, up to first order, as

$$
\mathbf{f}_{k}\left(\mathbf{x}_{k}\right) \cong \mathbf{f}_{k}\left(\hat{\mathbf{x}}_{k}\right)+\mathbf{F}_{k} \tilde{\boldsymbol{x}}_{k}
$$

where

$$
\mathbf{F}_{k}=\frac{\partial \mathbf{f}_{k}}{\partial \mathbf{x}_{k}}\left(\hat{\mathbf{x}}_{k}\right)
$$

equation $(28)$ is then approximated as

$$
\mathbf{f}_{k}\left(\hat{\mathbf{x}}_{k}\right)=\mathbf{x}_{k+1}-\mathbf{F}_{k} \tilde{\mathbf{x}}_{k}-\mathbf{w}_{k}
$$

The previous equation is regarded as a pseudo-measurement equation of $\mathbf{x}_{k+1}$, with the 'measurement' $\mathbf{f}_{k}\left(\hat{\boldsymbol{x}}_{k}\right)$ and the 'noise' $-\mathbf{F}_{k} \tilde{\mathbf{x}}_{k}-\mathbf{w}_{k}$ having the covariance matrix $\mathbf{F}_{k} \mathbf{P}_{k} \mathbf{F}_{k}^{T}+\mathbf{Q}_{k}$.

Moreover, the constraint equation (30) is also treated as a pseudo-measurement equation, with 'measurement' zero 
and no 'noise'. Equations (33), (29) and (30) can be then written in the form of $(5)$, with the entities

$$
\begin{aligned}
\mathbf{y} & =\left[\begin{array}{c}
\mathbf{f}_{k}\left(\hat{\mathbf{x}}_{k}\right) \\
\mathbf{z}_{k+1} \\
0
\end{array}\right], \quad \mathbf{h}\left(\mathbf{x}_{k+1}\right)=\left[\begin{array}{c}
\mathbf{x}_{k+1} \\
\boldsymbol{\Phi}_{k+1}\left(\mathbf{x}_{k+1}\right) \\
\mathbf{l}_{k+1}\left(\mathbf{x}_{k+1}\right)
\end{array}\right] \text { and } \\
\boldsymbol{\Gamma} \boldsymbol{\eta} & =\left[\begin{array}{c}
-\mathbf{F}_{k} \tilde{\mathbf{x}}_{k}-\mathbf{w}_{k} \\
v_{k+1} \\
0
\end{array}\right]
\end{aligned}
$$

The corresponding covariance matrix of the disturbances is

$$
\mathbf{S}_{k+1}=\left[\begin{array}{ccc}
\mathbf{F}_{k} \mathbf{P}_{k} \mathbf{F}_{k}^{T}+\mathbf{Q}_{k} & \mathbf{0} & \mathbf{0} \\
\mathbf{0} & \mathbf{R}_{k+1} & \mathbf{0} \\
\mathbf{0} & \mathbf{0} & \mathbf{0}
\end{array}\right]
$$

which is again singular. It is thus necessary to invoke the method discussed in Section 2.2 to find the maximumlikelihood estimate $\hat{\mathbf{x}}_{k+1}$ and the associated error covariance matrix.

In order to achieve quick convergence in the NewtonRaphson iterations, the estimate of the linearised system is first sought. The procedure outlined in Section 3.1 is applied to linear systems (22), (23) and (24), with

$\mathbf{A}_{k}=\mathbf{F}_{k}, \quad \mathbf{C}_{k+1}=\frac{\partial \mathbf{\Phi}_{k+1}}{\partial \mathbf{x}}\left(\mathbf{f}_{k}\left(\hat{\mathbf{x}}_{k}\right)\right), \quad \mathbf{L}_{k+1}=\frac{\partial \mathbf{l}_{k+1}}{\partial \mathbf{x}}\left(\mathbf{f}_{k}\left(\hat{\mathbf{x}}_{k}\right)\right)$

The solution is then used as the prior estimate for the nonlinear filter. With such a prefilter, the algorithm proposed here for designing a filter for constrained problems becomes quite efficient, as demonstrated by the example of target tracking discussed in the next Section.

\subsection{Filtering method for nonlinear system with soft constraints}

For some physical systems, the constraint conditions may not be exactly in the form of (30). Instead, the system governed by (28) may be subjected to soft constraints in the following form:

$$
\mathbf{l}_{k}\left(\mathbf{x}_{k}\right)=\mu_{k}
$$

Here $\mu_{k}$ denotes the random vectors with zero-mean and covariance matrix $\mathbf{T}_{k}$, representing the slackness of the constraints, which may come from the physical condition or from the approximation of the exact constraints.

To deal with such a soft constraint, we simply replace the covariance matrix $\mathbf{S}_{k+1}$ in (35) by the following form

$$
\mathbf{S}_{k+1}=\left[\begin{array}{ccc}
\mathbf{F}_{k} \mathbf{P}_{k} \mathbf{F}_{k}^{T}+\mathbf{Q}_{k} & \mathbf{0} & \mathbf{0} \\
\mathbf{0} & \mathbf{R}_{k+1} & \mathbf{0} \\
\mathbf{0} & \mathbf{0} & \mathbf{T}_{k+1}
\end{array}\right]
$$

If $\mathbf{T}_{k+1}$ is close to singular, the matrix $\mathbf{S}_{k+1}$ is close to singular as well and the use of the classical maximumlikelihood method may cause significant errors during the computation. However, the idea of the modified maximumlikelihood method discussed in Section 2.2 can be applied. The same procedure of the filtering method discussed in the previous Subsection can be adopted to perform filtering for such problems with soft constraints.

It is seen that the treatments of hard and soft constraints are essentially the same in our approach, no matter how close to singular the covariance matrix $\mathbf{T}_{k}$ is. However, in using the method of EKF with constraints such as the one in [4], a sequence of covariance matrices approaching $\mathbf{T}_{k}$ must be invoked, if $\mathbf{T}_{k}$ is almost singular. This observation shows one of the advantages of the methodology proposed in this paper.

\section{Example: target tracking problem with kinematic constraint}

To illustrate the proposed methodology, let us consider the tracking problem of a target moving with constant speed. Associated with different models, the constraint may be treatcd as being either inherent or supplemental. Moreover, if the speed of the target varies about a constant value, the constraint becomes soft. Simulation results show that the constrained filtering method performs well for all the cases.

\subsection{Problem description}

Consider a target moving in a circular orbit with a constant angular rate $\omega$. A radar tracker is used to obtain the measurements of the range, the azimuth, and the elevation of the target. Let $\mathbf{r}, \mathbf{v}$ denote the position and the velocity of the target, respectively. The nonlinear constraint of constant speed is given by

$$
|\mathbf{v}|^{2}=\text { constant }=v_{0}^{2}
$$

which is of the kinematic type. To describe the motion, we may choose $\mathbf{r}, \mathbf{v}$ to form the state vector $\mathbf{x}$. The state equation can be modelled as

$$
\dot{\mathbf{x}}=\mathbf{F} \mathbf{x}
$$

where

$$
\mathbf{F}=\left[\begin{array}{ll}
\mathbf{0} & \mathbf{1} \\
\mathbf{0} & \mathbf{\Omega}
\end{array}\right]
$$

in which the block matrix $\Omega$ is formed by the components of the known angular velocity $\omega=\left(\omega_{1}, \omega_{2}, \omega_{3}\right)$ as

$$
\boldsymbol{\Omega}=\left[\begin{array}{ccc}
0 & -\omega_{3} & \omega_{2} \\
\omega_{3} & 0 & -\omega_{1} \\
-\omega_{2} & \omega_{1} & 0
\end{array}\right]
$$

It is evident that the condition (39) can be deduced from equation (40), and hence the constraint is inherent to the dynamical system.

Let $\rho, \phi, \theta$ denote the range, the azimuth, and the elevation, respectively, of the target from the radar center. The measurement equation can be expressed as

$$
\boldsymbol{\Phi}(\mathbf{x})+v=\left[\begin{array}{l}
\rho \\
\phi \\
\theta
\end{array}\right]+v=\left[\begin{array}{c}
\sqrt{\left(x^{2}+y^{2}+z^{2}\right)} \\
\tan ^{-1} \frac{y}{x} \\
\tan ^{-1} \frac{-z}{x^{2}+y^{2}}
\end{array}\right]+v
$$

where $x, y, z$ are the components of $\mathbf{r}$ with respect to the reference frame, with the origin located at the radar and $x$-axis, $y$-axis, $z$-axis pointing to the north, the east, and downward, respectively.

With the inclusion of measurement noise $v$, the problem is to estimate the position $\mathbf{r}$ subject to the kinematic constraint (39), with both the measurement equation and the constraint equation being nonlinear. Hence, the constrained filtering method for nonlinear systems proposed in the previous Section can be adopted.

Similar problems have been discussed in $[4,5]$ by imposing a different but equivalent constraint (excepting the constant value):

$$
\mathbf{v} \cdot \mathbf{a}=0
$$


where a denotes the acceleration of the target. To accommodate such a constraint, we consider the PVA model by defining the state vector $\mathbf{x}^{a}=(\mathbf{r}, \mathbf{v}, \mathbf{a})$ which satisfies the following equation:

$$
\dot{\mathbf{x}}^{a}=\mathbf{F}^{a} \mathbf{x}^{a}
$$

where

$$
\mathbf{F}^{a}=\left[\begin{array}{ccc}
\mathbf{0} & \mathbf{I} & \mathbf{0} \\
\mathbf{0} & \mathbf{0} & \mathbf{1} \\
\mathbf{0} & \mathbf{0} & \mathbf{\Omega}
\end{array}\right]
$$

It is noted that condition (44) cannot be derived from the state equation (45), and hence the constraint is supplemental. With the same measurement equations (43), the filtering problem associated with such a constraint can also be solved by using the method discussed in Section 3.2 .

In reality, most targets would never move at a constant speed, although their motion may reside in a given flight envelope [8]. Such constraints may be treated as soft constraints. Here we consider the following condition:

$$
\mathbf{v} \cdot \mathbf{a}=\mu
$$

where the random variable $\mu$ with covariance $\mathbf{T}$ is included to represent the uncertainty in the physical adherence of the constraint [5], imposed on the state $\mathbf{x}^{a}$ with dynamics

$$
\dot{\mathbf{x}}^{a}=\mathbf{F}^{a} \mathbf{x}^{a}+\mathbf{G} \beta
$$

where

$$
\mathbf{G}=\left[\begin{array}{l}
0 \\
0 \\
\mathbf{I}
\end{array}\right]
$$

and the process noise $\beta$ represents the jitter disturbances with strength $\mathbf{Q}$. With the same radar measurement, the filtering problem can be solved by using the technique for soft constraints as developed in Section 3.3.

\subsection{Filtering algorithms and simulation results}

To apply the method discussed in Section 3 to system (40), the equation is first discretised as

$$
\mathbf{x}_{k+1}=\mathbf{A}_{k} \mathbf{x}_{k}, \quad \mathbf{A}_{k}=e^{\left(t_{k+1}-t_{k}\right) \mathbf{F}}
$$

With the corresponding measurement equation and constraint equations in (29) and (30) being expressed as, respectively,

$$
\mathbf{z}_{k}=\boldsymbol{\Phi}\left(\mathbf{x}_{k}\right)+v_{k}, \quad l_{k}\left(\mathbf{x}_{k}\right)=\mathbf{v} \cdot \mathbf{v}-v_{0}^{2}
$$

the constrained filtering method is now ready to be applied. By adopting the scenario used in [4, 5], the target is initially located at $(1000,0,-500)(\mathrm{m})$ with velocity $(-70.7,70.7,0)(\mathrm{m} / \mathrm{s})$. While maintaining a constant speed of $100(\mathrm{~m} / \mathrm{s})$ and a constant altitude of $500(\mathrm{~m})$, the target manoeuvres in a circular orbit with constant angular rate $0.2(\mathrm{rad} / \mathrm{s})$. Furthermore, it is assumed that the radar tracks the target at $20 \mathrm{~Hz}$ with the standard deviation of the range, azimuth, elevation measurements being $8(\mathrm{~m})$, 3 (mrad), 3 (mrad), respectively.

From Monte Carlo simulation, the root mean square errors (RMSE) obtained using the proposed constrained filtering method are shown in Fig. 1 (dotted line). Two other methods were also used to attack the same problem, the method of plain EKF and that of EKF with constraint [4]. The latter essentially uses a series of soft constraints in the form of (47) with decreasing $T$ to relax the rigidity of the constraint of constant speed. Comparing the dashed line (plain EKF) and the solid line (EKF with constraint) also shown in Fig. 1, consideration of the constraint indeed reduces the estimation errors. Nevertheless, the performance of the constrained filtering method (dotted line) is significantly better than the others.

Similar phenomenon appears in applying the filtering methods to the tracking problem using the PVA model (45) with supplemental constraint (44). The corresponding discrete equation can be shown in the form of (50) by replacing $\mathbf{A}_{k}$ with

$$
\mathbf{A}_{k}^{a}=e^{\left(t_{k+1}-t_{k}\right) \mathbf{F}^{a}}
$$

With the constraint function

$$
l_{k}\left(\mathbf{x}_{k}\right)=\mathbf{v} \cdot \mathbf{a}
$$
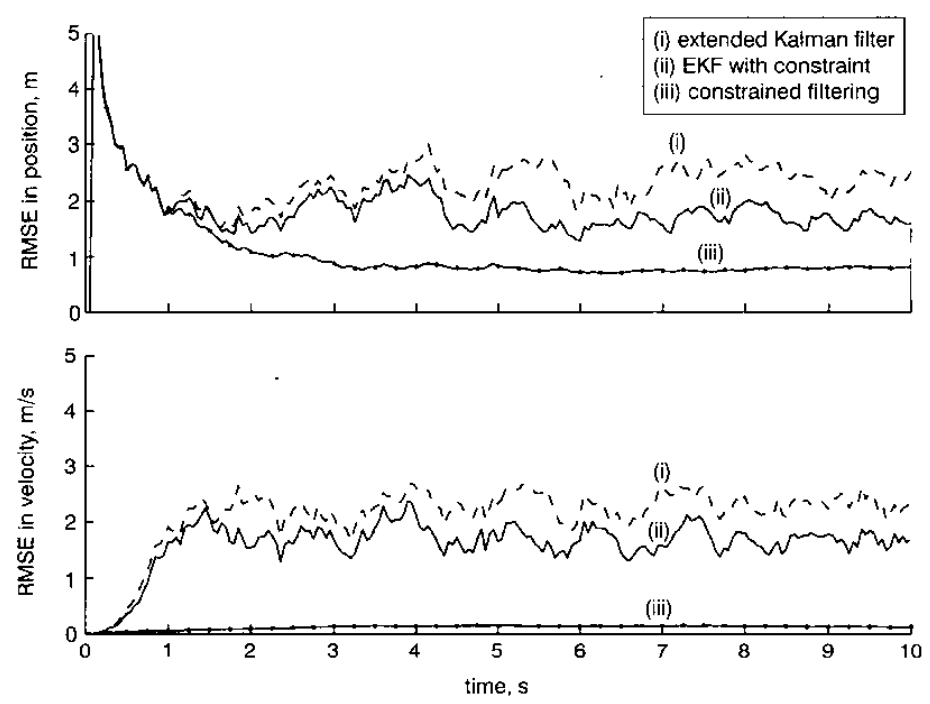

Fig. 1 RMSE estimation in $P-V$ model 


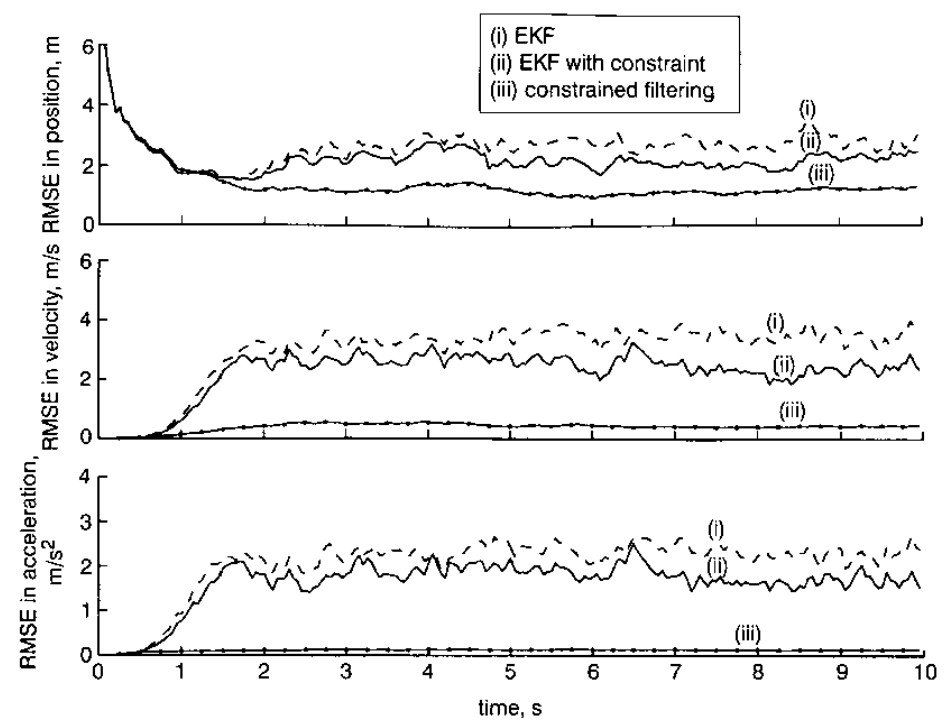

Fig. 2 RMSE estimation in P-V-A model

the constrained filtering method proposed in Section 3.2 is now applied. For the same scenario as in the previous case, the simulation results are shown in Fig. 2, along with the results of using the other two methods. The method developed in this paper again outperforms the others.

Now we consider the filtering problem with soft constraints. The discretisation of (48) is

$$
\mathbf{x}_{k+1}^{a}=\mathbf{A}_{k}^{a} \mathbf{x}_{k}^{a}+\mathbf{w}_{k}
$$

where

$$
\mathbf{w}_{k}=\int_{t_{k}}^{t_{k+1}} e^{\left(t_{k+1}-\tau\right) \mathbf{F}^{a}} \mathbf{G} \beta(\tau) d \tau
$$

and the covariance matrix for the disturbance $\mathbf{w}_{k}$ is given by

$$
\mathbf{Q}_{k}=\int_{t_{k}}^{t_{k+1}} e^{\left(t_{k+1}-\tau\right) \mathbf{F}^{u}} \mathbf{G} \mathbf{Q} \mathbf{G}^{T} e^{\left(t_{k+1}-\tau\right)\left(\mathbf{F}^{a}\right)^{T}} d \tau
$$

With the same measurement equation and the constrain function (53), the method proposed in Section 3.3 is used to estimate the motion of the target, with the process noise $\beta$ being assumed to be zero mean with variance $0.5\left(\mathrm{~m}^{2} / \mathrm{s}^{4}\right)$ in each axis and the slackness covariance matrix $\mathbf{T}_{k}$ being given by $\mathbf{I}$. Simulation results for this problem using different methods are shown in Fig. 3. It is seen that the proposed method is still better than the other two.

While the idea of treating constraint equation as pseudomeasurement has been used to deal with the target tracking problem in $[4,5]$, the method proposed in this paper is intrinsically different. For hard constraints, the conditions can be solved exactly, and for soft constraints, the algorithm works no matter how small the slackness of the constraint. For the constraint of a flight envelope, the idea of a particle filter is used in [8] to design an algorithm in which the stochastically gencrated samples or particles that

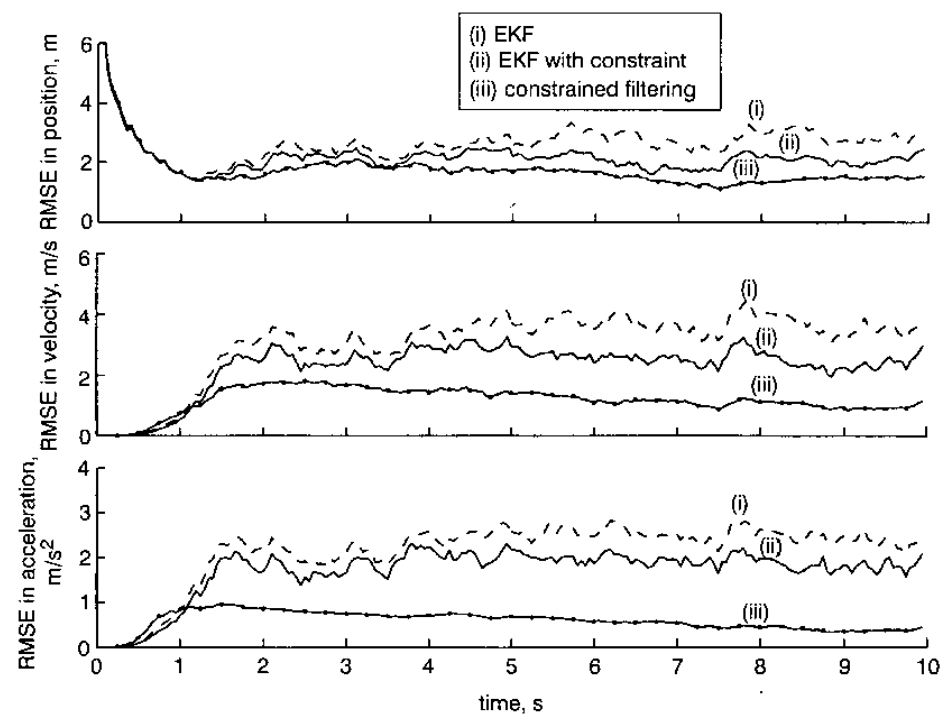

Fig. 3 RMSE estimation in P-V-A model with soft constraint 
are not in the flight envelope are discarded. While the performance is promising, as shown in [8], the computation issues make it difficult to implement the algorithm in real time [13]. Moreover, for the problem of dealing with either hard or soft constraints discussed in this paper, it is not conceivable to generate a suitable set of particles exactly satisfying the constraints to perform the estimation.

\section{Conclusion}

A constrained filtering method to tackle the filtering problems associated with nonlinear systems subject to constraint has been presented. By treating the system equations and constraint conditions as pseudo-measurements, and invoking the modified maximum-likelihood method to overcome the problems of nonlinearity and singularity, the proposed algorithm can successfully solve the problem, as illustrated by the example of target tracking. As described in Section 1, there are various types of constraints that exist for physical systems. Irrespective of whether they are inherent or supplemental, the conditions of constraint or the knowledge of the system characteristics should be taken into account in the filter design. To accommodate these constraints or information, linear or nonlinear, hard or soft, the methodology proposed in this paper provides an effective scheme to design an optimal filter as an alternative to the existing ones.

\section{References}

1 CAMPBELL, S.L.: 'Singular systems of differential equations' (Pitman, San Francisco, CA, 1980)

2 CAMPBELL, S.L.: 'Singular systems of differential equations II' (Pitman, San Francisco, CA, 1982)

3 NIKOUKHAH, R., WILLSKY, A.S., and LEVY, B.C.: 'Kalman filtering and Riccati equations for descriptor systems', IEEE Trans. Autom. Control, 1992, 37, (9), pp. 1325-1342

4 ALOUANI, A.T., and BLAIR, W.D.: 'Use of kinematic constraint in tracking constant specd, mancuvering targets', IEEE Trans. Autom. Control, 1993, 38, pp. 1107-1111

5 TAHK, M., and SPEYER, J.L.: 'Target tracking problems subject to kinematic constraints', IEEE Trans. Autom. Control, 1990, 38, pp. 324326

6 GEETER, J.D., BRUSSEL, H.V, and SCHUTTER, J.D.: 'A smoothly constrained Kalman filter', IEEE Trans. Pattern Anal Mach. Intell. 1997, 19, pp. 1171-1177

7 CHIANG, Y.T.. WANG, L.S., CHANG, F.R., and PENG, H.M.: "Constrained filtering method for attitude determination using GPS and Gyro', IEE Proc., Radar, Sonar, Navig., 2002, 149, (5), pp. 258-264

8 CHALla, S., ARULAMPALAM, S., and BERGMAN, N.: 'Target tracking incorporating flight envelope information'. Proceedings of the 3rd International Conference on Information fusion. FUSION 2000, 3rd International Confer
Paris, France, July 2000

9 BEST, R.A.: 'Integrated tracking and guidance'. PhD, School of Electronic and Electrical Engineering, University of Birmingham, Oct. 1996

10 CAMPBELL, S.L., and MEYER, C.D.: 'Generalized inverse of linear transforms' (Pitman, London, 1979)

11 LEON, S.G.: 'Linear algebra with applications' (Prentice Hall, Upper Saddle River, NJ, 2002, 6th edn.)

12 PENG, H.M., CHIANG, Y.T., CHANG, F.R., and WANG, L.S.: 'Maxmum-likelihood-based filtering for attitude determination via GPS carrier phase'. Proceedings of IEEE Position location and navigation Symposium, San Diego, California, March 2000, pp. 480-487

13 LIN, X., KIRUBARAJAN, T., BAR-SHALOM, Y,, and MASKELL, S.: 'Comparison of EKF, pseudomeasurement filter, and particle filter for a bearing-only target tracking problem', in DRUNNOND, O.E.(Ed.), Proc. SPIE, Signal and Data Processing of Small Targets 2002, Vol. 4728, pp. $240-250$ 